\title{
Table-Top Soft X-Ray Laser Interferograms of Dense Laser-Created Plasma
}

\author{
E. Jankowska, E. C. Hammarsten, J. Filevich, M. C. Marconi, and J. J. Rocca
}

\begin{abstract}
We report soft X-ray interferograms that illustrate the use of a very compact table-top soft $X$-ray laser in mapping the electron density evolution of a dense plasma. The spatial density distribution of a point-focus laser-created plasma with $\sim 300 \mu \mathrm{m}$ dimension was measured for densities up to $\sim 6.5 \times 10^{20} \mathrm{~cm}^{-3}$.
\end{abstract}

Index Terms-Capillary discharge lasers, plasma diagnostics, soft X-ray interferometry, soft X-ray lasers.

$\mathbf{T}$ HE DEVELOPMENT of soft X-ray lasers allows the extension of plasma interferometry to previously unreachable plasma parameter regions of high electron densities and large plasma lengths [1]. The advent of compact saturated soft $\mathrm{X}$-ray table-top lasers that can be fired at increased repetition rate [2], [3] in combination with new soft X-ray interferometers [4] now permits the application of this powerful plasma density diagnostics technique to the study of a variety of high-density plasmas. We have previously used a capillary discharge pumped table-top soft X-ray laser operating at $46.9 \mathrm{~nm}$ in combination with an amplitude division interferometer to study the evolution of a large-scale line-focus laser-created plasma [4]. This soft X-ray interferometer, that uses diffraction gratings as beam splitters, allows for significantly increased fringe visibility when compared to that obtained with the same laser source and a wavefront division interferometer based on Lloyd's mirror [5]. Interferograms of line-focus laser-created plasmas a few millimeters in length, with densities up to $5 \times 10^{19} \mathrm{~cm}^{-3}$ were previously reported.

Herein, we report soft X-ray interferograms that depict the evolution of a point-focus laser-created plasma. The electron density profiles constructed from the interferograms map plasma densities up to $\sim 6.5 \times 10^{20} \mathrm{~cm}^{-3}$ in regions where the plasma is about $300 \mu \mathrm{m}$ in diameter. The measurements correspond to $\mathrm{Cu}$ plasmas generated by focusing $0.62 \mathrm{~J}$ pulses from a Nd:YAG laser onto spots $<30 \mu \mathrm{m}$ diameter $(\sim 7$ $\mathrm{TW} / \mathrm{cm}^{2}$ ). The soft $\mathrm{X}$-ray laser used in this experiment is a very compact capillary discharge-pumped $\mathrm{Ne}$-like $\mathrm{Ar}$ amplifier that emits at a wavelength of $46.9 \mathrm{~nm}$ [2], corresponding to a

Manuscript received June 25, 2001. This work was supported in part by the U.S. Department of Energy under Grant DE-FG03-98DP00208, in part by the W. M. Keck Foundation, and in part by CONICET. The development of the soft $\mathrm{X}$-ray laser was supported by the National Science Foundation.

E. Jankowska is with the Department of Physics, Wroclaw University of Technology, Wroclaw 50-370, Poland.

E. C. Hammarsten, J. Filevich, and J. J. Rocca are with the Department of Electrical and Computer Engineering, Colorado State University, Fort Collins, CO 80523 USA (e-mail: rocca@engr.colostate.edu).

M. C. Marconi is with the Physics Department, Facultad de Ciencias Exactas y Naturales, Universidad de Buenos Aires, Buenos Aires C1428EHA, Argentina.

Publisher Item Identifier S 0093-3813(02)03310-6.
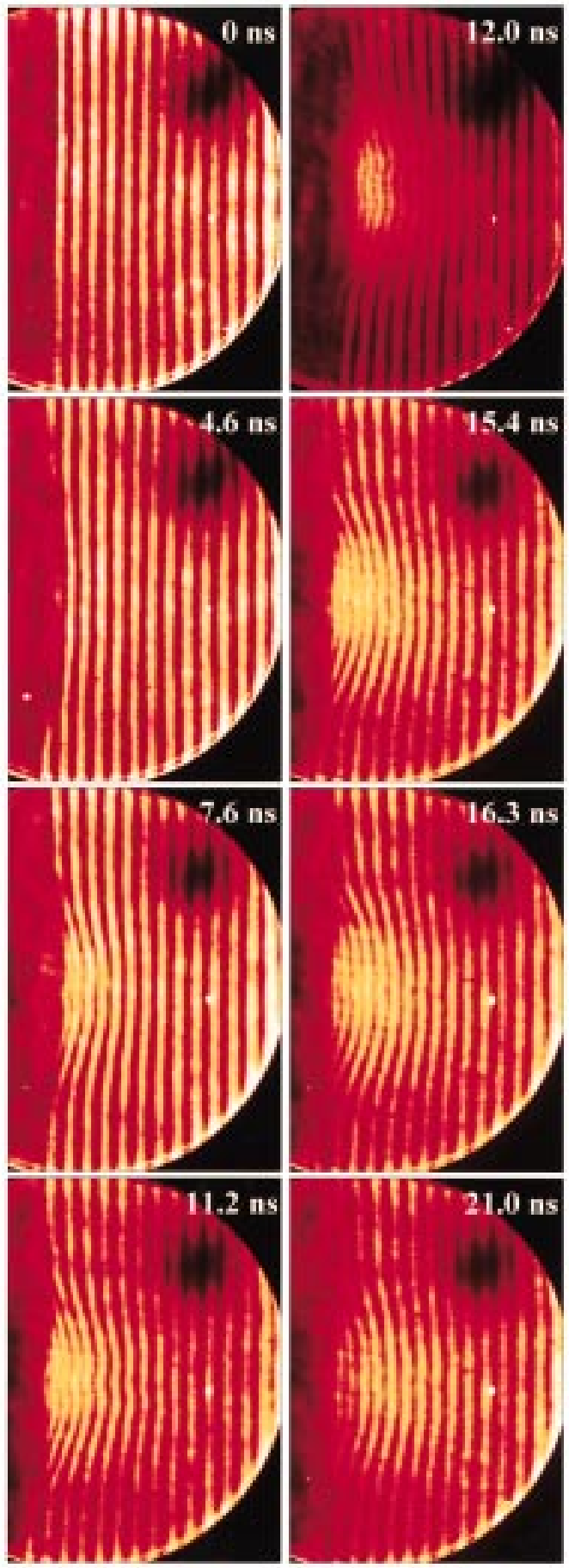

Fig. 1. Series of soft X-ray interferograms depicting the evolution of the laser-created plasma. The times are measured with respect to the initiation of the Nd:YAG laser pulse.

critical density of $n_{c}=5 \times 10^{23} \mathrm{~cm}^{-3}$. The measurements 


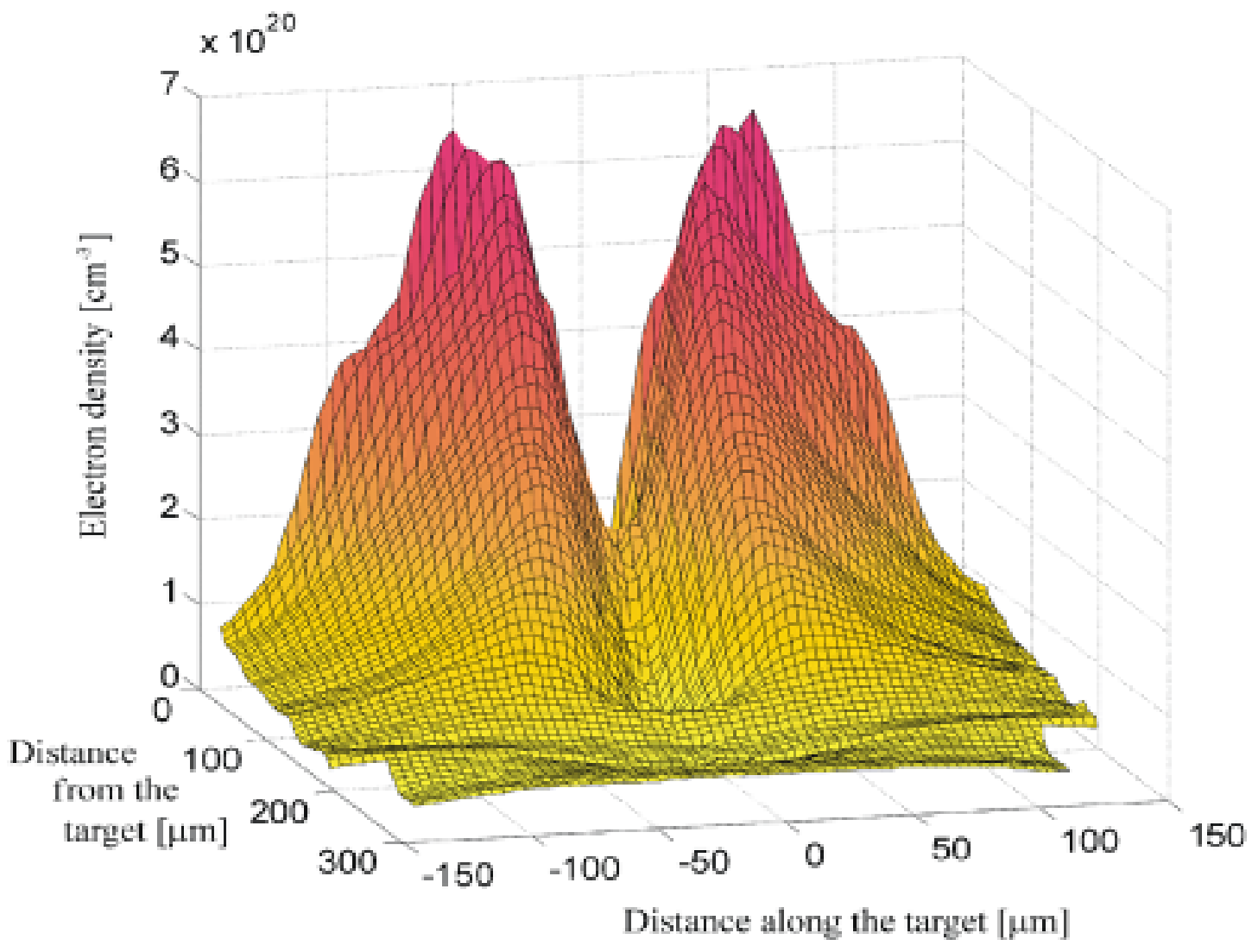

Fig.2. Electron density profile computed by an Abel inversion of the 12.0-ns interferogram.

were conducted using X-ray laser pulses of approximately $0.1 \mathrm{~mJ}$ and $1.2 \mathrm{~ns}$. A description of the diffraction grating amplitude division interferometer used in the experiment was presented in a recent publication [4]. The plasma was imaged with 51.2X magnification into a gated soft X-ray sensitive detector composed of a microchannel-plate, a phosphor screen, and a charged coupled device (CCD) array.

Fig. 1 shows a series of interferograms corresponding to fifth shots of the Nd:YAG laser on the same target location, a situation in which the observed plasma emanates from a $300-400-\mu \mathrm{m}$-deep hole drilled by the previous four shots. The electron density evolution of the plasma was mapped firing the capillary discharge soft X-ray laser at different time delays with respect to the beginning of the $\mathrm{Nd}$ :YAG laser pulse. Abel-inversion [6] of the measured fringe shift reveals electron density distributions such as that in Fig. 2, in which the electron density is plotted as a function of distance from the target. This electron density profile corresponds to an interferogram obtained $12.0 \mathrm{~ns}$ after the initiation of the laser pulse, the time at which the electron density is observed to reach a maximum value of $\sim 6.5 \times 10^{20} \mathrm{~cm}^{-3}$ at a distance of $\sim 24 \mu \mathrm{m}$ from the target plane. A pronounced density depression, caused by the interaction of the high-density $\mathrm{Nd}$ :YAG laser beam with the expanding plasma is observed along the propagation axis of the laser beam. Comparison of such measurements with hydrodynamic code simulations can contribute to an improved understanding of the dynamics of high-density plasmas.

\section{REFERENCES}

[1] L. B. Da Silva, T. W. Barbee Jr., R. Caubre, P. Celliers, D. Ciarlo, S. Libby, R. A. London, D. Matthews, S. Mrowka, J. C. Moreno, D. Ress, J. E. Trebes, A. S. Wan, and F. Weber, "Electron density measurements of high density plasmas using soft X-ray laser interferometry," Phys. Rev. Lett., vol. 74, no. 20, pp. 3991-3994, 1995.

[2] C. D. Macchietto, B. R. Benware, and J. J. Rocca, "Generation of millijoule-level soft X-ray laser pulses at 4-Hz repetition rate in a highly saturated table-top capillary discharge amplifier," Opt. Lett., vol. 24, pp. 1115-1117, 1999

[3] Y. Li, J. Dunn, J. Nilsen, T. W. Barbee Jr., A. L. Osterheld, and V. N. Shlyaptsev, "Saturated tabletop X-ray system at $19 \mathrm{~nm}$, , J. Opt. Soc. Amer. B, Opt. Phys., vol. B17, pp. 1098-1101, 2000.

[4] J. Filevich, K. Kanizay, M. C. Marconi, J. L. A. Chilla, and J. J. Rocca, "Dense plasma diagnostics with an amplitude division laser interferometer based on diffraction gratings," Opt. Lett., vol. 25, pp. 356-359, 2000.

[5] J. J. Rocca, C. H. Moreno, M. C. Marconi, and K. Kanizay, "Soft X-ray laser interferometry of a plasma with a table-top laser and Lloyd's mirror," Opt. Lett., vol. 24, pp. 420-422, 1999.

[6] R. N. Bracewell, Fourier Transform and Its Applications. New York: McGraw-Hill, 1965. 